\title{
Implicación de la inhibición cognitiva en el gasto financiero en jóvenes de México. Estudio preliminar en neuroeconomía
}

\author{
Involvement of cognitive inhibition in financial spending \\ in young people of Mexico. Preliminary study in Neuroeconomics
}

Jorge Raúl Palacios ${ }^{1}$ y Karen Pescina Ortega ${ }^{2}$

${ }^{1} \mathrm{PhD}$ en psicología sobre modelos neurocognitivos. Investigador de tiempo completo en la Red de Investigación del Comportamiento del Consumidor de la Universidad del Valle de México,

Querétaro.E-mail: drjpalacios81@gmail.com

${ }^{2}$ Estudiante de Psicología en la Universidad del Valle de México, Querétaro.

E-mail: kpescina99@gmail.com

Universidad del Valle de México, Querétaro, México.

\section{Resumen}

La psicología y las neurociencias ofrecen nuevas perspectivas para comprender el comportamiento económico y del consumidor. La neuroeconomía provee un nuevo marco de referencia para comprender la forma en la que las estructuras neuroanatómicas del cerebro se encuentran implicadas en las decisiones financieras de los individuos. En este contexto, el objetivo de la investigación es identificar la asociación entre la inhibición cognitiva y la cantidad de dinero que gastan los universitarios. Participaron 40 jóvenes universitarios, $47.5 \%$ hombres y $52.5 \%$ mujeres, entre 18 y 25 años, de la Ciudad de Querétaro, México. La inhibición cognitiva se evaluó con el Test de Stroop. Además, se preguntó sobre datos sociodemográficos y los gastos mensuales. Los resultados muestran descriptivamente el nivel de gasto de los jóvenes y las puntuaciones del efecto Stroop. Los jóvenes tienen puntuaciones medias en Palabra, Color, Palabra Color e Interferencia, acorde a los parámetros establecidos. Respecto al gasto mensual se observa que, en promedio, gastan $\$ 666$ pesos mexicanos (34.04 USD / 31.13 EUR). Adicionalmente, se encontró un déficit en el control inhibitorio relacionado con un aumento en el nivel de gasto realizado por los estudiantes universitarios, es decir, aquellos que gastan y compran, en mayor medida presentan dificultades en su inhibición cognitiva (autocontrol). El control inhibitorio se asoció con la edad y esta, a su vez, con el nivel de dinero gastado. Por último, se profundiza en la utilidad de los resultados dentro de la neuroeconomía en México y demás países latinoamericanos, así como su aporte como línea de investigación. Palabra clave: neuroeconomía, Stroop test, decisiones financieras, inhibición cognitiva, universitarios.

\section{Abstract}

Psychology and neuroscience offer new perspectives to understand consumer and economic behaviour. Neuroeconomics provides a new frame of reference to understand the way in which the neuroanatomical structures of the brain are involved in the financial decisions of people. Currently, measurement scales are an application alternative to assess people's executive functions and cognitive inhibition quickly. In the present study for the evaluation of cognitive inhibition 
we will use the Stroop Test (Golden, 1994), which examines the cognitive processes associated with cognitive flexibility; the interference resistance from external stimuli and its effect on behaviour. The plates that make up the Stroop test present different possibilities for analysis and interpretation. Within neuroeconomics, there is an area that investigates how cognitive and affective regulation is critical to achieve economic results, in addition to investigating the problems caused by consumption and spending that people have in their daily lives. There is research shown that individuals who make compulsive purchases show cognitive deficiencies and difficulties in inhibiting their responses. Furthermore, compulsive purchases correlate with financial executive functioning, particularly with impulse control, organization and planning, showing that compulsive purchases are not limited only to impulse control. In Latin America and particularly in Mexico, there are few studies on neuroeconomics or consumer neuroscience, so consider that this research is an innovative contribution to the subject in our region. In this context, the objective of the research is to identify the association between cognitive inhibition and the money university students spend. This research evaluated the association between cognitive inhibition and the money university students spend. A total of 40 university students participated, $47.5 \%$ men and $52.5 \%$ women, between 18 and 25 years, from the City of Querétaro, Mexico. Cognitive inhibition was evaluated with the Stroop Test. In addition, sociodemographic data and monthly expenses were asked. The results descriptively detailed the level of youth spending and the Stroop effect variables. Young people have mean scores in Word, Colour, Word - Colour and Interference, according to the established parameters. Regarding monthly expenses, it is observed that on average they spend \$ 666 Mexican pesos (34.04 USD / 31.13 EUR). In addition, a deficit was found in the inhibitory control related to an increase in the level of expen- diture made by university students, that is, those who spend and buy more have difficulties in their cognitive inhibition (self-control). Inhibitory control is associated with age and this time with the level of money spent. Additionally, the inhibition processes allows us to assume certain activity in the orbitofrontal cortex (OFC) and the dorsolateral prefrontal cortex (DLFC), while they are ready to spend and who has problems with the control of their money, they cannot realize the excessive expenses that it does not recognize the symptoms that drive it to compulsive buying. The findings provide evidence to the neuroeconomy, as well as to the posture of cognitive control. Furthermore, it is corroborated that performance tests are an alternative in the detection of executive functioning deficiencies in a short time of application and provide evidence in the approach to neuroeconomics through this form of measurement. This offers a novel vision to understand the personal finances of Mexicans and their economic behaviour. Financial and consumer behaviour has become our main line of research from a neuroeconomic perspective; in the future, we intend to continue this study by incorporating affective and neurobehavioral factors involved in financial decisions in young people of our country. Finally, the usefulness of the results within the neuroeconomics in Mexico and Latin America is deepened, as well as its contribution as a line of research.

Keywords: neuroeconomics, Stroop test, financial decisions, cognitive inhibition, university.

\section{Introducción}

En la actualidad, la psicología y las neurociencias ofrecen nuevas perspectivas para comprender el comportamiento económico, dentro del que se destaca el comportamiento del consumidor y las decisiones financieras (Riveros, Arias y Pérez-Acosta, 2008), que incluyen el gasto, las compras, el ahorro o la inversión y constituyen una parte fundamental 
de las acciones que las personas realizan en la vida cotidiana (Palacios y Soler, 2017).

Desde la perspectiva de la economía conductual (Barberis y Thaler, 2003; Soler, Bustos, Palacios, Zeelenberg y Díaz-Loving, 2019; Thaler, 2016), se ha planteado que el comportamiento económico comprende las acciones, los deseos y los pensamientos a la hora de decidir entre gasto o ahorro (Prieto y Aznal, 2006). La literatura sobre los determinantes del comportamiento financiero (Barros, Denegrí y Valenzuela, 2019; Coria, Toro y López, 2007; Mitchell y Lusardi, 2015; Ortega y Rodríguez-Vargas, 2005) encuentran relaciones entre la educación financiera y las características sociodemográficas de los individuos, y destacan que las personas con mayores niveles de educación financiera planean mejor, ahorran más, ganan más en sus inversiones y manejan mejor su dinero para el retiro. En cuanto a los estudios sobre los gastos, Denegrí, Barros, Cárdenas, Sepúlveda y Vivallo (2017) encuentran que los adolescentes de Chile utilizan su dinero principalmente en gastos personales $(46.2 \%)$, de ocio $(30.8 \%)$ y gastos de alimentación (23.1 $\%$ ). Respecto al endeudamiento, los estudiantes solicitan préstamos de dinero para cubrir necesidades de alimentación (56\%), transporte $(33 \%)$ y vestimenta $(33 \%)$.

Por otra parte, el estudio de la neuroeconomía unifica la economía, la psicología y las neurociencias, incorporando diferentes técnicas o mediciones, así como una variedad de constructos que permiten comprender las diferencias individuales con efectos directos en la vida social de las personas (Declerck, Boone y Emonds, 2013; Fehr y Camerer, 2007; Kahneman y Frederick, 2002). La neuroeconomía (Camerer, Loewenstein y Prelec, 2005; Kenning y Plassmann, 2005; Sebastian, 2014; Zak, 2004) provee un nuevo marco de referencia para comprender la forma en la que las estructuras neuroanatómicas del cerebro se encuentran implicadas en las decisiones de los individuos (Glimcher y Rustichini, 2004; Loewenstein, Rick y Cohen, 2008; Sanfey,
Loewenstein, McClure y Cohen, 2006; San Martín, Isla y Melis, 2012).

Es importante destacar que en la investigación sobre neuroeconomía y la neurociencia del consumidor, se utiliza resonancia magnética funcional (fMRI) en el procesamiento de la información de las áreas del cerebro que se activan en la compra de productos o en las decisiones financieras. Adicionalmente, existen diversos instrumentos como el electroencefalograma (EEG) o los potenciales evocados que se utilizan en estudios experimentales para analizar la compra de productos o las decisiones financieras (Lavín, San Martín, Bravo, Contreras e Isla, 2011; San Martín, Kwak, Pearson, Woldorff y Huettel, 2016), especialmente de investigadores latinoamericanos que han presentado estudios en la toma de decisiones con evidencia experimental.

Sin embargo, desde otra perspectiva de medición, existen instrumentos psicométricos con base neuroconductual para valorar el funcionamiento estructural y ejecutivo del cerebro (Anderson, 2002; Gioia, Isquith, Retzlaff y Espy, 2002; Pedrero, Ruiz, Rojo, Morales, Pedrero, Lorenzo y González, 2016; Spinella, 2005). Por lo tanto, su aplicación se convierte en una alternativa novedosa para la detección de posibles déficits del funcionamiento ejecutivo en diferentes contextos, particularmente en los que se requiere un breve tiempo de aplicación para evaluar las funciones ejecutivas de las personas.

Las funciones ejecutivas constituyen un conjunto de procesos que intervienen en el control del comportamiento, el pensamiento, así como en las emociones, para cumplir objetivos en situaciones nuevas o complejas (Baggetta, y Alexander, 2016; Bernal, Rodríguez y Ortega, 2020; Miyake, Friedman, Emerson, Witzki y Howerter, 2000) y que se encuentran asociadas neuroanatómicamente a diferentes circuitos neuronales en los lóbulos frontales del córtex (Ambrosini, Arbula, Rossato, Pacella y Valles, 2019; Happaney, Zelazo y Stuss, 2004; Jódar, 2004). 
Dentro de los procesos del funcionamiento ejecutivo, el control inhibitorio es uno de sus principales componentes, ya que permite a las personas regular una respuesta o una reacción inmediata, al esperar o ejecutar una respuesta, retrasando la gratificación, inhibiendo la primera reacción impulsiva o sustituirla por una respuesta adaptada al contexto en el que se desenvuelve (Lin, Liew y Pérez, 2019). La función principal de la inhibición consiste en el control de las tendencias ligadas al pensamiento, al comportamiento y a los estímulos ambientales que interfieren con el logro de las tareas y objetivos actuales (Aydmune y Introzzi, 2018).

Por otro lado, se ha descripto que la actividad en el área frontal del cerebro se evalúa con tareas clásicas de función ejecutiva (Verdejo, Orozco, Meersmans y Pérez, 2004; Willer, Tiso, Haider, Hinds, Baker, Miecznikowski y Leddy, 2018). En el presente estudio, para la evaluación de la inhibición cognitiva se utilizó el Test de Stroop Colores y Palabras (Golden, 1994), que examina los procesos cognitivos asociados con la flexibilidad cognitiva; la resistencia de interferencia procedente de estímulos externos y su efecto sobre la conducta. Las láminas que integran la prueba Stroop presentan posibilidades de análisis e interpretaciones diferentes. La lámina $\mathrm{P}$ (listado de palabras en tinta negra) y la lámina $\mathrm{C}$ (listado de palabras con equis, con tinta de colores) son sensibles al lenguaje, es decir que se observa la velocidad con la que la persona lee en 45 segundos la aplicación. En la última lámina PC (palabras presentadas con diferentes colores), se evalúa la capacidad de la persona para inhibir una respuesta automática a favor de otras más inusuales, también conocido como el efecto Stroop (Rodríguez, Pineda y del Carmen, 2016). Así, se verifica la flexibilidad cognitiva, la inhibición en la respuesta automática y el control atencional (Martín, Hernández, Rodríguez, García, Díaz y Jiménez, 2015).

MacLeod (1991) reporta que los objetos y los colores toman más tiempo al nombrarlos en voz alta que solo leer palabras. Lo crucial en este proceso está dado por la presencia de la información interferente, la cual debe ser inhibida para lograr completar la tarea requerida. En la lámina de interferencia se requiere tanto de los niveles de atención necesarios como de la capacidad inhibitoria de la respuesta automática de manera sostenida (Rodríguez et al., 2016). De manera consecuente, en el Stroop color - palabra, la tarea del estímulo está diseñada de tal manera que se evoca tanto la respuesta como la interferencia cognitiva de forma que no puede ser disociada, lo cual produce el efecto de inhibición de la respuesta automática. La capacidad inhibitoria de la interferencia permite que la inhibición de una respuesta automática responda de una manera adecuada a un determinado estímulo al encontrarse con los componentes de dirección de la atención y la selección de los estímulos presentados (Gutiérrez-Martínez, Ramos-Ortega y Vila-Chaves, 2018; MacLeod, 1998).

La diversidad de estímulos ante los cuales las personas tienen que responder puede llevar a la formación de sesgos cognitivos en su capacidad para tomar decisiones (MacLeod y MacDonald, 2000). De acuerdo con Killikelly y Szücs (2010), la tarea importante a realizar y la interacción de la tarea irrelevante a las características del estímulo causan un efecto de interferencia. La interferencia en esta tarea es medida con el decremento en el tiempo de reacción o la exactitud en las condiciones incongruentes. La mayoría de estudios que exploran los procesos de inhibición utilizando variantes del paradigma Stroop muestran un incremento de la actividad en áreas frontales en la condición de interferencia en la región orbitofrontal derecha (Tirapu, Muñoz, Pelegrín y Albéniz, 2005).

La investigación sobre fallas inhibitorias se encuentra en varios comportamientos, como el Trastorno por Déficit de Atención e Hiperactividad -TDAH- (Krieger, Amador-Campos y Gallardo-Pujol, 2019) o la depresión (Crandall, Allsop y Hanson, 2018). Además, los problemas inhibitorios se asocian a compor- 
tamientos que presentan dificultades en el control de impulsos y que son nocivos para la salud como las adicciones (Holmes, Hollinshead, Roffman, Smoller y Buckner, 2016; Martins, Bartholow, Cooper, Von Gunten y Wood, 2018). Adicionalmente, una disminución cognitiva asociada con la edad se explica por un déficit vinculado al funcionamiento inhibitorio (Rognoni, Casals-Coll, SánchezBenavides, Quintana, Manero, Calvo, Palomo, Aranciva, Tamayo y Peña-Casanova, 2013; Wecker, Kramer, Wisniewski, Delis y Kaplan, 2000). Dentro de la neuroeconomía existe un área que investiga cómo la regulación cognitiva y afectiva es crítica para lograr la satisfacción de resultados económicos además de indagar los problemas causados con el consumo y el gasto que tienen las personas en su vida diaria (Hubert, 2010). Al respecto, Plassmann, O'Doherty y Rangel (2007) investigaron las bases neurales de la disposición a pagar en transacciones de alimentos y encontraron que la actividad en la corteza orbitofrontal (OFC), la corteza anterior cingulada y la corteza prefrontal dorsolateral (DLFC) se asocia positivamente con la disposición de pago y que la corteza medial codifica el valor de las transacciones económicas. Schaefer (2009) sugiere que las estructuras asociadas con el circuito de recompensa (estriado) y el área dorsolateral de la corteza prefrontal pueden estar involucradas en la preferencia de marca y, por lo tanto, decidir comprarla.

Otros estudios (Derbyshire, Chamberlain, Odlaug, Schreiber y Grant, 2014) han señalado que los individuos que realizan compras compulsivas muestran deficiencias cognitivas y dificultades en la inhibición de sus respuestas. Spinella, Yang y Lester (2007) demuestran que las compras compulsivas correlacionan con el funcionamiento ejecutivo financiero, en particular, con el control de impulsos, la organización y la planeación, evidenciando que las compras compulsivas no se limitan únicamente al control de los impulsos. Quien presenta problemas con las compras compulsivas no puede percatarse de los gastos exce- sivos y tampoco reconoce los síntomas que lo impulsan a la compra compulsiva (Rodríguez, Saucedo, Hernández y Gutiérrez, 2016).

En personas compradoras compulsivas, Raab, Elger, Neuner y Weber (2011) encontraron actividad en la corteza prefrontal en el área ventromedial y dorsolateral al momento de comprar productos, por lo que, además, poseen menos autocontrol y mayor depresión. Posteriormente, Kühn, Strelow y Gallinat (2016) pronosticaron el comportamiento del consumidor en un supermercado, basados en imágenes de resonancia magnética funcional (fMRI). Recopilaron los datos mientras los participantes veían anuncios de barra de chocolate y fotos de productos, antes y después de cada uno. Los datos de fMRI se extrajeron de regiones cerebrales seleccionadas a priori: núcleo accumbens, corteza orbitofrontal medial, amígdala, hipocampo, circunvolución frontal inferior y corteza prefrontal dorsomedial, que contribuyen positivamente, y la corteza prefrontal dorsolateral y la ínsula, que contribuyen negativamente a las ventas. Los cambios en las ventas se pronosticaron mediante la señal de fMRI durante la visualización del anuncio $\mathrm{y}$, en segundo lugar, mediante una comparación de la señal cerebral durante la visualización del producto antes y después del anuncio.

Por otra parte, en el contexto mexicano, existen pocos estudios que describan el perfil del comportamiento económico de los jóvenes, sus hábitos como consumidores $\mathrm{y}$ el de uso del dinero que tienen. Adicionalmente, los problemas relacionados con la obtención de ingresos, la falta de educación financiera y el manejo del dinero son los que más se presentan en este grupo de edad. Por ejemplo, el $87 \%$ no tiene ahorro para el retiro (Encuesta de Cultura Financiera de los jóvenes en México [ECF], 2014) y se ha reportado (Asociación Mexicana de la Industria Publicitaria y Comercial en Internet [AMIPCI], 2018) que los usuarios en México compran cada mes algún producto en línea, con un gasto promedio trimestral de sus compras de $\$$ 
6300 pesos mexicanos (322.30 USD / 294.39 EUR).

A pesar de que en México se ha estudiado el comportamiento del consumidor como una subdivisión de la psicología financiera, aún se carece de estudios que identifiquen las decisiones que toman las personas respecto de sus finanzas. Considerando la investigación sobre la elección financiera, las finanzas conductuales proveen un marco de referencia para comprender las decisiones que toman las personas respecto de su comportamiento económico. Particularmente, dentro del enfoque de la neuroeconomía o de la economía conductual, en nuestro país, Palacios y Bustos (2019) describieron la gratificación financiera y la aversión a la pérdida que tienen hombres y mujeres, así como el nivel de gasto desde la elección intertemporal y la aversión al riesgo financiero. Encuentran que hombres y mujeres toman la elección de pagar de contado y prefieren las ganancias a los riesgos; las mujeres presentan un riesgo moderado y los hombres invierten con mayor riesgo financiero.

Por otra parte, el estudio de la inhibición cognitiva sobre el gasto de los jóvenes es relevante, porque estos se encuentran en una etapa antecedente al ámbito laboral, lo que permitiría obtener información referente del manejo de su dinero y, asimismo, su apertura a la posibilidad de contar con precursores de las potenciales deudas que podrán adquirir en la etapa adulta. Debido a esto, se podrían proponer acciones concretas en sus finanzas personales tomando en consideración los factores neurocognoscitivos implicados en la toma de decisiones financieras, el comportamiento de consumo y el nivel de gasto de los jóvenes.

A partir de lo anterior, se decidió investigar el procesamiento cognitivo, basado en los patrones de inhibición. Se optó por utilizar el efecto Stroop, por ser una medida del funcionamiento ejecutivo para determinar la capacidad para inhibir o controlar una respuesta dominante (Gutiérrez-Martínez et al., 2018). En Latinoamérica, y en particular en México, son escasos los estudios sobre neuroeconomía o neurociencia del consumidor, por lo que consideró que la presente investigación es un aporte innovador a la temática en la región. En este sentido, en este equipo de investigación, desde hace algunos años, se ha comenzado a investigar cómo las personas toman decisiones con su dinero a partir de correlatos neuroconductuales. Por tal motivo, el objetivo de la investigación fue identificar el nivel de asociación entre la inhibición cognitiva (efecto Stroop) y la cantidad de dinero que gastan los jóvenes. Se planteó probar que los jóvenes que gastan más dinero tendrán dificultades en su control inhibitorio.

\section{Método}

\section{Participantes}

Se seleccionó una muestra no probabilística de forma intencionada de 40 jóvenes, 47.5 $\%$ hombres y $52.5 \%$ mujeres, con un rango de edad entre 18 y 25 años, con una media de $20.20(\mathrm{DE}=1.6)$ años. El $94.9 \%$ son solteros y el $5.1 \%$ vive en unión libre. Todos eran estudiantes universitarios de la Ciudad de Querétaro, México.

\section{Instrumento}

\section{Test de Stroop}

Se aplicó el instrumento de Golden (1994), en su versión española, el cual se encuentra integrado por tres secciones, cada una de ellas constituida por 100 estímulos. La primera sección (Palabra / P) está formada por cuatro columnas con 20 palabras (las palabras utilizadas son "rojo", "verde" y "azul") ordenadas al azar e impresas con tinta negra, que se presentan a cada persona. Se solicitó al participante la lectura en voz alta de todas las palabras. En el apartado dos (Color / C), se encuentra conformado por grupos de cuatro equis ("XXXX"); cada grupo está impreso en color azul, verde o rojo. Los colores no 
siguen el mismo orden en la hoja. La actividad a realizar por los participantes consiste en nombrar el color de la tinta de tantos ítems como sea posible. Por último, la tercera sección (Palabra - Color / PC) se compone por las palabras de los colores que aparecen en la sección 1, coloreados con los colores de tinta de la sección 2, combinados de tal forma que la palabra escrita y el color de la tinta son incongruentes. Se solicitó al participante que nombre el color de la tinta, inhibiendo la lectura de la palabra. En cada sección, la tarea propuesta duró 45 segundos para cada lámina.

\section{Datos demográficos}

Se integró una sección impresa que incluía datos como el sexo, edad, estado civil, ciudad en la que vive y una pregunta referente a sus gastos mensuales.

\section{Procedimiento}

En primer lugar, se solicitó permiso para realizar el estudio a las autoridades correspondientes y se invitó a los estudiantes a colaborar en la investigación. Se explicó que el estudio estaba relacionado con actividades que realizan en su vida cotidiana. Se les solicitó que respondieran de forma sincera, y se les explicó que sus respuestas se utilizarían únicamente para fines de investigación.

Posteriormente, a cada estudiante se le aplicó la prueba Stroop de manera individual, en un tiempo aproximado de 10 minutos. Para cada uno de ellos, se utilizó un manual con las mismas hojas y una extra en la que se colocaron los resultados de cada uno. El orden de presentación de las hojas fueron: palabra $(\mathrm{P})$ para evaluar la velocidad con la que pueden leer las palabras, color (C) para nombrar el color y palabra - color (PC) para evaluar el funcionamiento ejecutivo enfocado en la inhibición cognitiva. Antes de la aplicación de la prueba, se les entregó la hoja de consentimiento informado y un cuestionario con datos demográficos.

\section{Consideraciones éticas}

A cada participante se le pidió su colaboración, mediante la firma de un formato de consentimiento y asentimiento informado. Se les aclaró que la información era anónima, se les garantizó la confidencialidad de los datos proporcionados y se resolvieron las dudas que tuvieran con respecto a su participación. El protocolo de investigación fue establecido de acuerdo al Reglamento de la Ley General de Salud en su apartado sobre investigación en seres humanos (Secretaría de Salud, 2011). El proyecto de investigación se encuentra evaluado por el Comité de Bioética y registrado ante el Comité de Investigación de la Universidad del Valle de México, Campus Querétaro, con el número de registro CSUVMI2017-002.

\section{Análisis de datos}

Los análisis de datos se realizaron considerando la transformación de las puntuaciones del efecto Stroop. Se realizó un análisis descriptivo (medidas de tendencia central y dispersión) para los indicadores del efecto Stroop y el nivel de gasto. Posteriormente, se efectuó un análisis de correlación de Rho de Spearman entre la inhibición (Palabra, Color, Palabra - Color, e interferencia), la edad y el gasto mensual reportado por los participantes de la investigación, mediante el programa SPSS 14.

\section{Resultados}

En la Tabla 1 se aprecia que los jóvenes tienen puntuaciones medias en P, C, PC e Int acorde a los parámetros establecidos. Respecto al gasto mensual, se observa que en promedio gastan \$ 666 pesos mexicanos (34.04 USD / 31.13 EUR) y que el gasto máximo que llegan a tener es de $\$ 3000$ pesos mexicanos (153.33 USD / 140.21 EUR). 
Tabla 1

Descripción de la inhibición cognitiva y el gasto mensual.

\begin{tabular}{c|c|c|c|c} 
& Media & Desviación Estándar & Mínimo & Máximo \\
\hline Palabra & 109.70 & 15.0 & 73.0 & 140.0 \\
Color & 76.70 & 12.7 & 53.0 & 110.0 \\
Palabra - Color & 49.40 & 10.2 & 25.0 & 82.0 \\
Interferencia & 4.41 & 8.6 & -11.2 & 34.02 \\
Errores & .875 & 1.1 & 1.0 & 4.0 \\
Gasto mensual & 666.75 & 626.8 & 200 & 3000
\end{tabular}

Se encontró que la edad se asoció de forma negativa y significativa con el color / C $(\mathrm{r}=$ $-.382 ; \mathrm{p}<.05)$ y la palabra - color / PC $(\mathrm{r}=$ -.495; $\mathrm{p}<.01$ ), con una magnitud moderada. Adicionalmente, la edad se relacionó positiva, significativamente y con una magnitud fuerte con el nivel de gasto $(r=.555 ; \mathrm{p}<.001)$.

La Tabla 2 muestra que la palabra, el color y la palabra - color presentan correlaciones positivas, moderadas y estadísticamente significativas. En cuanto al nivel de gasto que realizan los jóvenes, se encontraron correlaciones negativas, altas y significativas con $\mathrm{PC}$, interferencia y $\mathrm{C}$. El nivel de gasto tiene la correlación más alta con $\mathrm{PC}$, lo que permite asumir que es la variable más cercana con el nivel de gasto que realizan los jóvenes en el último mes. No se encontraron relaciones significativas con $\mathrm{P}$ y errores.

Tabla 2

Correlaciones entre los indicadores del efecto Stroop y el gasto mensual.

\begin{tabular}{c|c|c|c|c|c} 
& Color & Palabra - Color & Interferencia & Errores & Gasto mensual \\
\hline Palabra / P & $.479^{* *}$ & $.442^{* *}$ & -.186 & $-.440^{* *}$ & -.129 \\
Color / C & & $.445^{* *}$ & -.199 & $-.465^{* *}$ & $-.348^{*}$ \\
Palabra- Color / PC & & & $.513^{* *}$ & $-.481^{* *}$ & $-.526^{* *}$ \\
Interferencia / Int & & & & .069 & $-.377^{*}$ \\
Errores & & & & & .130
\end{tabular}

${ }^{*} \mathrm{p}<.05 ; * * \mathrm{p}<.01 ; * * * \mathrm{p}<.001$

\section{Discusión}

En el artículo se ha reportado una de las primeras investigaciones sobre neuroeconomía en México. El estudio tuvo como objetivo relacionar la inhibición cognitiva mediante el efecto Stroop con la cantidad de dinero que gastan los jóvenes. Dentro de los principales hallazgos, se encontró que un déficit en el control inhibitorio estaría relacionado con un aumento en el nivel de gasto realizado por los estudiantes universitarios.
Esta asociación sugiere que entre más dinero gasten los estudiantes, presentarán una mayor deficiencia en el control inhibitorio.

Al analizar descriptivamente el nivel de gasto, los resultados obtenidos permiten señalar que en los participantes del estudio el gasto mínimo y el promedio son cantidades por debajo del promedio reportado por estudios similares (Palacios y Bustos, 2019) y solamente las cantidades máximas de gasto se equiparan con el promedio de gasto comparado contra el mismo estudio. Los descu- 
brimientos encontrados respecto del nivel de gasto son relevantes, ya que permiten contar con un seguimiento del nivel de gasto que tienen los universitarios e identificar un posible perfil respecto a sus finanzas personales. En futuros estudios se podrá indagar en qué gastan (Asociación Mexicana de la Industria Publicitaria y Comercial en Internet [AMIPCI], 2018; ECF, 2014) o en qué se endeudan estos jóvenes, como se ha reportado en estudios previos (Denegrí et al., 2017).

Al considerar descriptivamente las puntuaciones del test de Stroop en esta muestra de jóvenes universitarios, se puede mencionar que los resultados obtenidos en la investigación son ligeramente superiores en $\mathrm{P}, \mathrm{C} \mathrm{y}$ $\mathrm{PC}$ a los reportados en muestras de Colombia (Rodríguez et al., 2016), es decir que estos universitarios de México en comparación con los de Colombia, en promedio, logran identificar un mayor número de palabras, colores $\mathrm{y}$ estímulos. Al comparar los datos con muestras de españoles, se puede observar que los datos se ubican en el promedio de lo reportado con estudios previos (Golden, 1994). Al momento de analizar los datos promedio del índice de Interferencia, se observa nuevamente puntuaciones más elevadas que los colombianos, y calificaciones promedio y similares a los españoles. En otras palabras, según la puntuación obtenida en la tarea de interferencia estimada, se puede observar que, en promedio, los jóvenes universitarios de México inhiben correctamente las respuestas a los estímulos presentados. En relación con la edad, se observa que los jóvenes de menor edad mencionan más colores y suprimen mediante control voluntario la respuesta automática de lectura de palabras (MacLeod, 1991, 1998; Martín et al., 2015).

Al considerar el nivel de asociación entre la inhibición cognitiva y el gasto semanal de los jóvenes, se encontró como resultado principal una correlación negativa con color $(\mathrm{C})$, Palabra - Color (PC) e Interferencia (Int), es decir que, entre más dinero gastan semanalmente, menor inhibición cognitiva, lo cual evidencia un peor control de la interferencia en esta etapa de su formación. La capacidad de resistir la interferencia ejercida por la lectura parece estar presente entre este grupo de edad. La evidencia encontrada sostiene la hipótesis planteada que postula que los jóvenes que gastan más dinero tendrán dificultades en su control inhibitorio.

Por otra parte, los estudios sobre funciones ejecutivas (Anderson, 2002; Baggetta y Alexander, 2016; Happaney et al., 2004; Willer et al., 2018) describen que estas actúan de forma concertada para guiar el comportamiento hasta conseguir una meta, cumplir con una tarea y autorregular la conducta. La inhibición es una de las funciones principales, encaminada al control del pensamiento, el comportamiento y los estímulos ambientales que interfieren con el logro de tareas y objetivos actuales (Ambrosini et al., 2019; Aydmune y Introzzi, 2018; Verdejo et al., 2004). Dentro de la inhibición cognitiva e interferencia, al existir un decremento en el tiempo de reacción, las personas regulan una respuesta o reacción inmediata al esperar o ejecutar una respuesta, lo cual retrasas la gratificación e inhibe la primera reacción impulsiva al sustituirla por una respuesta adaptada al contexto en el que se desenvuelve (Lin et al., 2019), tal como sucede con los jóvenes que presentan un menor nivel de gasto.

Considerar el funcionamiento ejecutivo como responsable de la inhibición cognitiva invita a pensar el rol que esta posee para que un joven controle respuestas automáticas, cambiando de una respuesta a otra sin dificultades en su ejecución, lo que da como resultado la inhibición del comportamiento ajustado al contexto social en el que se desenvuelve en su vida diaria. Particularmente, en esta investigación hace referencia al manejo del dinero, su gasto y las compras que puede realizar un joven universitario, de forma que si la inhibición tiene una influencia en la conducta (Gutiérrez-Martínez, Ramos-Ortega y Vila-Chaves, 2018) también se encontrará asociada en el control financiero (Palacios y 
Soler, 2017) y el nivel de gasto de los jóvenes de nuestro entorno. La evidencia encontrada demuestra que los jóvenes que tienen un gasto mayor presentan dificultades en su inhibición cognitiva, lo cual parece ir en concordancia con los estudios (Palacios y Bustos, 2019; Prieto y Aznal, 2006; Rodríguez et al., 2016; Spinella et al., 2007) que mencionan que la falta de autocontrol se asocia con el gasto y la compra no responsable en los jóvenes.

Los resultados encontrados respecto al nivel de gasto en los universitarios evidencia la importancia de establecer estrategias para diferentes segmentos poblacionales en función de sus capacidades financieras, por ejemplo, los jóvenes, las mujeres, las personas con menores niveles de educación, las personas de bajos ingresos y quienes no tienen capacidad para ahorrar (Mitchell y Lusardi, 2015; Riveros et al., 2008). Sin lugar a dudas, un tema pendiente por analizar es la brecha de género, por las diferencias entre el gasto y los ingresos entre los hombres y mujeres. Aunque esta brecha no afecta a todas las mujeres por igual, es importante reflexionar sobre las implicaciones en la inclusión y las políticas financieras en México y en el resto de países latinoamericanos, ya que las mujeres que participan en la toma de decisiones financieras de sus hogares pueden desarrollar mejores capacidades financieras. Por lo tanto, la existencia de programas que busquen promover el empoderamiento femenino a través de la inclusión de actividades empresariales o de emprendimiento puede también favorecer las conductas y actitudes de las familias para mejorar su bienestar financiero (Ortega y Rodríguez-Vargas, 2005; Palacios y Soler, 2017).

Estas y otras directrices pueden ofrecer alternativas para el desarrollo de productos financieros, especialmente a través de mecanismos formales como cuentas de ahorro, que tienen un impacto sobre las capacidades financieras de los individuos (Barberis y Thaler, 2003; Palacios y Bustos, 2019). Los programas de inclusión y educación financiera deben enfocarse no solamente en la transmisión de conceptos y conocimientos, sino también incidir en actitudes relacionadas con el ahorro y el gasto. La promoción de productos financieros innovadores por medio de la banca por internet y que se ajusten a las necesidades de los diferentes segmentos poblacionales permitirá mejorar las decisiones financieras de los individuos (Barros et al., 2019; Coria et al., 2007; Denegrí et al., 2017; ECF, 2014; Prieto y Aznal, 2006).

Por otra parte, de forma paralela se apoya la propuesta (Derbyshire et al., 2014; Raab et al., 2011; Spinella et al., 2007) de quienes señalan que los individuos que realizan compras compulsivas muestran deficiencias cognitivas, dificultades en la inhibición de sus respuestas y en el control de impulsos, y poseen menos autocontrol. Adicionalmente, los procesos de inhibición permiten asumir cierta actividad en la corteza orbitofrontal (OFC) y la corteza prefrontal dorsolateral (DLFC) mientras se disponen a gastar (Kühn et al., 2016; Plassmann, et al., 2007), y quien presenta problemas con el control de su dinero no puede percatarse de los gastos excesivos que realiza, así como tampoco reconoce los síntomas que lo impulsan a una compra compulsiva.

De acuerdo con los resultados obtenidos en las asociaciones entre la edad, el efecto Stroop y el nivel de gasto, se puede mencionar que a mayor edad, hay menos control de gasto $\mathrm{y}$, asimismo, hay una disminución cognitiva asociada a la edad en C y PC. Lo anterior puede explicarse, en parte, por un déficit vinculado al funcionamiento inhibitorio (Wecker et al., 2000) que las personas estarían atravesando en esta etapa de la vida (Rognoni et al., 2013).

Los hallazgos encontrados aportan evidencia a la neuroeconomía (Loewenstein et al., 2008; Sanfey et al., 2006; Zak, 2004), así como a la postura del control cognitivo (Camerer et al., 2005; Kahneman y Frederick, 2002), con lo que se corrobora que las pruebas de ejecución (Gioia et al., 2002; Pedrero-Pérez et al., 2016; Spinella, 2005) son una alterna- 
tiva en la detección de deficiencias del funcionamiento ejecutivo en un breve tiempo de aplicación y aportan evidencia en el abordaje de la neuroeconomía a través de esta forma de medición. Lo anterior ofrece una visión novedosa para comprender las finanzas personales de los mexicanos (Palacios y Soler, 2017; Palacios y Bustos, 2019) y su comportamiento económico.

La principal limitación que contiene la investigación es que no se preguntó sobre los ingresos de los participantes, lo que hubiera permitido distinguir con mayor precisión su comportamiento al momento de gastar, así como indagar en qué realizan sus gastos. Estudios posteriores deberán obtener los datos de ingreso, para considerarse dentro de los análisis. La segunda limitante es el tamaño de la muestra, que no es representativa de la población universitaria, además de no haberse realizado de forma probabilística; por lo tanto, los resultados de este estudio no se pueden generalizar para toda la población juvenil. Una tercera limitación del estudio fue no contar con un espacio definido para realizar la prueba Stroop, por lo que se tuvo que realizar en tres escenarios diferentes, lo que pudo influir en no controlar algunos distractores como el ruido al momento de responder el test de Stroop. A pesar de esta limitante, se considera que los hallazgos encontrados son una primera aportación de la neuroeconomía en Latinoamérica, principalmente en México. Para mejorar el estudio, se propone utilizar un Stroop computarizado con la finalidad de agilizar las aplicaciones, así como incorporar un Stroop emocional sobre el dinero para identificar su efecto sobre la toma de decisiones financiera y del consumidor.

Para finalizar, hay que señalar que el comportamiento financiero y del consumidor se ha constituido en la principal línea de investigación bajo una perspectiva neuroeconómica. En este sentido, para dar continuidad al presente estudio, se tendrá en cuenta, a futuro, incorporar factores afectivos y neuroconductuales involucrados en las decisiones financieras de los habitantes de México.

En conclusión, los hallazgos de esta investigación sobre la asociación entre la inhibición cognitiva (efecto Stroop) y la cantidad de dinero que gastan los jóvenes comprobaron que los jóvenes que gastan más dinero tienen dificultades de inhibición. Los jóvenes mexicanos de este estudio en promedio pueden identificar un mayor número de palabras, colores, así como inhibición de estímulos, comparados con datos de otras muestras. Además, los resultados indican que, a medida que los jóvenes aumentan de edad, se incrementa su nivel de gasto.

\section{Referencias}

Ambrosini, E., Arbula, S., Rossato, C., Pacella, V. y Valles, A. (2019). Neuro-cognitive architecture of executive functions: A latent variable análisis. Cortex, 119, 441-456. https://doi. org/10.1016/j.cortex.2019.07.013

AMIPCI (2018). Estudio Comercio Electrónico en México 2017. http://asociaciondeinternet. org.mx/es/component/remository/Comercio-Electronico/Estudio-de-Comercio-Electronico-en-Mexico-2016/lang,es-es/?Itemid=

Anderson, P. (2002). Assessment and development of executive function (EF) during childhood. Child Neuropsychology, 8(2), 71-82. https:// doi.org/10.1076/chin.8.2.71.8724

Aydmune, Y. e Introzzi, I. (2018). Inhibición: una función ejecutiva difícil de medir. Algunas problemáticas en relación con las pruebas de inhibición informatizadas. Psicodebate. Psicología, Cultura y Sociedad, 18(2), 7-25. https:// doi.org/10.18682/pd.v18i2.741

Baggetta, P. y Alexander, P. A. (2016). Conceptualization and operationalization of executive function. Mind, Brain, and Education, 10(1), 10-33. https://doi.org/10.1111/mbe. 12100

Barberis, N. y Thaler, R. (2003). A survey of behavioral finance. Handbook of the Economics of Finance, 1, 1053-1128. https:// doi.org/10.1016/S1574-0102(03)01027-6

Barros, B., Denegrí, C. M. y Valenzuela, P. S. (2019). Consumo, actitudes hacia el endeuda- 
miento, materialismo e influencia de pares en adolescentes rurales del sur de Chile. Interdisciplinaria, 36(1), 203-219. https://doi. org/10.16888/I.V36I1.593

Bernal, R., Rodríguez, V. M. y Ortega, A. (2020). Estimulación de las funciones ejecutivas y su influencia en el rendimiento académico en escolares de primero básico. Interdisciplinaria, 37(1), 1-34. https://doi.org/10.16888/ interd.2020.37.1.6

Camerer, C., Loewenstein, G. y Prelec, D. (2005). Neuroeconomics: How neuroscience can inform economics. Journal of Economic Literature, 43(1), 9-64. https://doi. org/10.1257/0022051053737843

Coria, M. D., Toro, G. M. y López, S. E. (2007). La comprensión del funcionamiento bancario en adolescentes chilenos: un estudio de psicología económica. Interdisciplinaria, 24(2), 137-159. https://www.redalyc.org/pdf/180/18024201. pdf

Crandall, A., Allsop, Y. y Hanson, C. L. (2018). The longitudinal association between cognitive control capacities, suicidality, and depression during late adolescence and young adulthood. Journal of Adolescence, 65, 167-176. https://doi.org/10.1016/j.adolescence.2018.03.009

Declerck, C. H., Boone, C. y Emonds, G. (2013). When do people cooperate? The neuroeconomics of prosocial decision making. Brain and cognition, 81(1), 95-117. https://doi. org/10.1016/j.bandc.2012.09.009

Denegrí, C. M., Barros, S., Cárdenas C., Sepúlveda A. J. y Vivallo, U. O. (2017). Consumo y endeudamiento en adolescentes escolarizados de la Región de la Araucanía, Chile. Estudios Pedagógicos, 43(1), 61 - 74. https://doi. org/10.4067/S0718-07052017000100004

Derbyshire, K. L., Chamberlain, S. R., Odlaug, B. L., Schreiber, L. y Grant, J. E. (2014). Neurocognitive functioning in compulsive buying disorder. Annals of Clinical Psychiatry, 26(1), 57-63. https://www.ncbi.nlm.nih.gov/ pubmed/24501731

Encuesta de Cultura Financiera de los jóvenes en México (ECF). (2014). Síntesis de resultados.
Banamex- UNAM. https://www.banamex. com/es/conoce_banamex/quienes_somos/ prensa/pdf/book_brujula_digital_2014.pdf

Fehr, E. y Camerer, C. F. (2007). Social neuroeconomics: the neural circuitry of social preferences. Trends in Cognitive Sciences, 11(10), 419-427. https://doi.org/10.1016/j. tics.2007.09.002

Gioia, G. A., Isquith, P., Retzlaff, P. y Espy, K. A. (2002). Confirmatory factor analysis of the Behavior Rating Inventory of Executive Function (BRIEF) in a Clinical Sample. Child Neuropsychology, 8(4) 249- 257. https://doi. org/10.1076/chin.8.4.249.13513

Glimcher, P. W. y Rustichini, A. (2004). Neuroeconomics: the consilience of brain and decision. Science, 306(5695), 447-452. https://doi. org/10.1126/science. 1102566

Golden, C. J. (1994). Stroop: Test de colores y palabras: Manual. Madrid: TEA Ediciones.

Gutiérrez-Martínez, F., Ramos-Ortega, M. y Vila-Chaves, J. O. (2018). Eficacia ejecutiva en tareas de interferencia tipo Stroop. Estudio de validación de una versión numérica y manual (CANUM). Anales de Psicología, 34(1), 184-196. https://doi.org/10.6018/ analesps.34.1.263431

Happaney, K., Zelazo, P.H. y Stuss, D. T. (2004). Development of orbitofrontal function: Current themes and future directions. Brain and Cognition, 55, 1-10. https://doi.org/10.1016/j. bandc.2004.01.001

Holmes, A. J., Hollinshead, M. O., Roffman, J. L., Smoller, J. W. y Buckner, R. L. (2016). Individual differences in cognitive control circuit anatomy link sensation seeking, impulsivity, and substance use. Journal of Neuroscience, 36(14), 4038-4049. https://doi. org/10.1523/JNEUROSCI.3206-15.2016

Hubert, M. (2010). Does neuroeconomics give new impetus to economic and consumer research? Journal of Economic Psychology, 31(5), 812-817. https://doi. org/10.1016/j.joep.2010.03.009

Jódar, V. M. (2004). Funciones cognitivas del lóbulo frontal. Revista de Neurología, 39, 178182. https://doi.org/10.33588/rn.3902.2004254 
Kahneman, D. y Frederick, S. (2002). Representativeness revisited: Attribute substitution in intuitive judgment. En T. Gilovich, D. Griffin y D. Kahneman (Eds.), Heuristics and biases: The psychology of intuitive judgment ( $p$. 49-81). Cambridge University Press. https:// doi.org/10.1017/CBO9780511808098.004

Kenning, P. y Plassmann, H. (2005). Neuroeconomics: an overview from an economic perspective. Brain Research Bulletin, 67(5), 343-354. https://doi.org/10.1016/j.brainresbull.2005.07.006

Killikelly, C. y Szücs, D. (2010). The development of interference control: A pilot study using the manual colour word stroop paradigm. Procedia: Social and Behavioral Sciences, 2(2), 4842-4847. https://doi.org/10.1016/j. sbspro.2010.03.781

Krieger, V., Amador-Campos, J. A. y Gallardo-Pujol, D. (2019). Temperament, executive function, and attention-deficit/hyperactivity disorder (ADHD) in adolescents: The mediating role of effortful control. Journal of Clinical and Experimental Neuropsychology, 41(6), 615-633. https://doi.org/10.1080/13803395.20 19.1599824

Kühn, S., Strelow, E. y Gallinat, J. (2016). Multiple "buy buttons" in the brain: Forecasting chocolate sales at point-of-sale based on functional brain activation using fMRI. NeuroImage, 136, 122-128. https://doi.org/10.1016/j.neuroimage.2016.05.021

Lavín, C., San Martín, R., Bravo, D., Contreras, S. e Isla, P. (2011). Potenciales cerebrales relacionados a feedback en el estudio del aprendizaje y la toma de decisiones económicas. Revista Latinoamericana de Psicología, 43(3), 455-471. https://doi.org/10.14349/rlp.v43i3.220

Lin, B., Liew, J. y Pérez, M. (2019). Measurement of self-regulation in early childhood: Relations between laboratory and performance-based measures of effortful control and executive functioning. Early Childhood Research Quarterly, 47(2), 1-8. https://doi.org/10.1016/j. ecresq.2018.10.004

Loewenstein G., Rick S. y Cohen J. D. (2008). Neuroeconomics. Annual
Review of Psychology, 59, 647-672. https://doi.org/10.1146/annurev. psych.59.103006.093710

MacLeod, C. M. (1991). Half a century of research on the Stroop effect: An integrative review. Psychological Bulletin, 109(2), 163-203. https://doi.org/10.1037/0033-2909.109.2.163

MacLeod, C. M. (1998). Training on integrated versus separated Stroop tasks: The progression of interference and facilitation. Memory \& Cognition, 26, 201-211. https://doi. org/10.3758/bf03201133

MacLeod, C. M. y MacDonald, P. A. (2000). Interdimensional interference in the Stroop effect: Uncovering the cognitive and neural anatomy of attention. Trends in Cognitive Sciences, 4 (10), 383-391. https://doi.org/10.1016/s13646613(00)01530-8

Martín, R., Hernández, S., Rodríguez, C., García, E., Díaz, A. y Jiménez, J. E. (2015). Datos normativos para el Test de Stroop: patrón de desarrollo de la inhibición y formas alternativas para su evaluación. European Journal of Education and Psychology, 5(1), 39- 51. https://doi.org/10.30552/ejep.v5i1.76

Martins, J. S., Bartholow, B. D., Cooper, M. L., Von Gunten, C. D. y Wood, P. K. (2018). Associations between executive functioning, affect-regulation drinking motives, and alcohol use and problems. Psychology of Addictive Behaviors, 32(1), 16- 28. https://doi. org/10.1037/adb0000324

Mitchell, O. S. y Lusardi, A. (2015). Financial literacy and economic outcomes: Evidence and policy implications. The Journal of Retirement, 3(1), 107-114. https://doi.org/10.3905/ jor.2015.3.1.107

Miyake, A., Friedman, N. P., Emerson, M. J. Witzki, A. y Howerter, A. (2000). The unity and diversity of executive functions and their contributions to complex "Frontal Lobe" tasks: A latent variable analysis. Cognitive Psychology, 41, 49- 100. https://doi.org/10.1006/ cogp.1999.0734

Ortega, V. y Rodríguez-Vargas, J. C. (2005). Consumo y deuda en adultos jóvenes: evaluación desde un modelo integrador de la conducta 
económica. Revista Latinoamericana de Psicologia, 37(1), 95-118. https://www.redalyc.org/ pdf/805/80537106.pdf

Palacios, J.y Bustos,A.M.(2019). Decisionesfinancieras y aversión al riesgo desde una perspectiva de la economía conductual, Nthe, 26, 18-25. http://nthe.mx/archivos/revista26.php

Palacios, J. y Soler, A. L. (2017). Preliminary study of the comparative profile of financial control in university young people. Ciencias Psicológicas, 11(2), 201-212. https://doi. org/10.22235/cp.v11i2.1491

Pedrero-Pérez, E. J., Ruiz- Sánchez de León, J. M., Rojo-Mota, G., Morales- Alonso, S., Pedrero Aguilar, J., Lorenzo, I. y González, Á. (2016). Inventario de Síntomas Prefrontales (ISP): validez ecológica y convergencia con medidas neuropsicológicas. Revista de Neurologia, 63(6), 241-251.https://doi.org/10.33588/ rn.6306.2016143

Plassmann, H., O’Doherty, J. y Rangel, A. (2007). Orbitofrontal cortex encodes willingness to pay in everyday economic transactions. Journal of Neuroscience, 27(37), 9984-9988. https://doi. org/10.1523/JNEUROSCI.2131-07.2007

Prieto, J. G. y Aznal, A. S. (2006). Influencia de la ansiedad en la adicción al consumo y la falta de autocontrol en la compra y el gasto de los jóvenes. Estudios sobre Consumo, 76, 43-57. https://www.researchgate.net/profile/ Alejandro_Salcedo2/publication/28108823 Influencia_de_la_ansiedad_en_la_adiccion_ al_consumo_y_la_falta_de_autocontrol en_la_compra_y_el_gasto_de_los_jovenes/ links/0c96053bfa1be4f239000000/Influenciade-la-ansiedad-en-la-adiccion-al-consumo-yla-falta-de-autocontrol-en-la-compra-y-el-gasto-de-los-jovenes.pdf

Raab, G., Elger, C. E., Neuner, M. y Weber, B. (2011). A neurological study of compulsive buying behaviour. Journal of Consumer Policy, 34(4), 401. https://doi.org/10.1007/ s10603-011-9168-3

Riveros, A. A., Arias, P. R. y Pérez-Acosta, A. M. (2008). Psicología y asuntos económicos: una aproximación al estado del arte. International Journal of Psychological Research, 1(1),
49-57. https://doi.org/10.21500/20112084.965

Rodríguez, B. L., Pineda, R. C. y del Carmen, P. N. (2016). Propiedades psicométricas del Stroop, test de colores y palabras en población colombiana no patológica. Universitas Psychologica, 15(2), 255-272. https://doi. org/10.11144/Javeriana.upsy15-2.ppst

Rodríguez, G. M., Saucedo S. J., Hernández, B., A. y Gutiérrez, C. O. (2016). Análisis del comportamiento de compra para identificar compradores compulsivos en Saltillo, Coahuila, México. Revista Internacional Administración \& Finanzas, 5(5), 27-44. https://ssrn.com/ abstract $=2916395$

Rognoni, T., Casals-Coll, M., Sánchez-Benavides, G., Quintana, M., Manero, R. M., Calvo, L., ... y Peña-Casanova, J. (2013). Estudios normativos españoles en población adulta joven (proyecto NEURONORMA jóvenes): normas para las pruebas Stroop Color-Word Interference Test y Tower of London-Drexel University. Neurología, 28(2), 73-80. https://doi. org/10.1016/j.nrl.2012.02.009

Sanfey, A. G., Loewenstein, G., McClure, S. M. y Cohen, J. D. (2006). Neuroeconomics: cross-currents in research on decision-making. Trends in Cognitive Sciences, 10, 108-116. https://doi.org/10.1016/j. tics.2006.01.009

San Martín, R., Isla, P. y Melis, C. (2012). Preferencia temporal en el cerebro: Una revisión crítica de las contribuciones de la neuroeconomía al estudio de la elección intertemporal. El Trimestre Económico, 79(314), 449-473. https://doi.org/10.20430/ete.v79i314.65

San Martín, R., Kwak, Y., Pearson, J. M., Woldorff, M. G. y Huettel, S. A. (2016) Altruistic traits are predicted by neural responses to monetary outcomes for self versus charity. Social Cognitive And Affective Neuroscience, 11(6), 863-876. https://doi.org/10.1093/scan/nsw026

Secretaría de Salud. (2011). Reglamento de la Ley General de Salud en Materia de Investigación para la Salud. www.salud.gob.mx/unidades/ cdi/nom/compi/rlgsmis.html

Sebastian, V. (2014). New directions in understanding the decision-making process: Neuro- 
economics and neuromarketing. Procedia-Social and Behavioral Sciences, 127, 758-762. https://core.ac.uk/download/pdf/81197172.pdf

Soler, A. L., Bustos, A. M., Palacios, J., Zeelenberg, M. y Díaz-Loving, R. (2019). Emotional and rational purchasing decisions in spending: Creation and validation of Purchase Decision-Making Inventory (PDMI). Suma Psicológica, 26 (2), 75-85. https://doi. org/10.14349/sumapsi.2019.v26.n2.3

Spinella, M. (2005). Self-rated executive function development of the executive function index. International Journal of Neuroscience, 115, 649-667. https://doi. org/10.1080/00207450590524304

Spinella, M., Yang, B. y Lester, D. (2007). Prefrontal systems in financial processing. The Journal of Socio-Economics, 36, 480- 489. https://doi.org/10.1016/j.socec.2006.12.008

Schaefer, M. (2009). Neuroeconomics: in search of the neural representation of brands. Progress in Brain Research, 178, 241-252. https:// doi.org/10.1016/S0079-6123(09)17817-2

Thaler, R. H. (2016). Behavioral economics: past, present, and future. American Economic Review, 106(7), 1577-1600. https://doi. org/10.1257/aer.106.7.1577

Tirapu, U., Muñoz, C. J., Pelegrín, V. C. y Albéniz, F. A. (2005). Propuesta de un protocolo para la evaluación de las funciones ejecutivas. Revista de Neurología, 41(3), 177- 186. https://doi. org/10.33588/rn.4103.2005054

Verdejo, A., Orozco, G. C., Sánchez, J., Aguilar, A. F. y Pérez, G. M. (2004). Impacto de la gravedad del consumo de drogas sobre distintos componentes de la función ejecutiva. Revista de Neurología, 38(12), 1109-1106. https://doi. org/10.33588/rn.3812.2003592

Wecker, N. S, Kramer, J.H., Wisniewski, A., Delis, D. C. y Kaplan, E. (2000). Age effects on executive ability. Neuropsychology, 14, 409-14. https://doi.org/10.1037/0894-4105.14.3.409

Willer, B. S., Tiso, M., Haider, M. N., Hinds, A. L., Baker, J. G., Miecznikowski, J. C. y Leddy, J. J. (2018). Evaluation of executive function and mental health in retired contact sport athletes. The Journal of Head Trauma Rehabilitation, 33(5), E9- E15. https://doi. org/10.1097/HTR.0000000000000423

Zak, P. J. (2004). Neuroeconomics. Biological Sciences, 359(1451), 1737-1748. https://doi. org/10.1098/rstb.2004.1544

Recibido: 16 de junio de 2020 Aceptado: 18 de octubre de 2021 